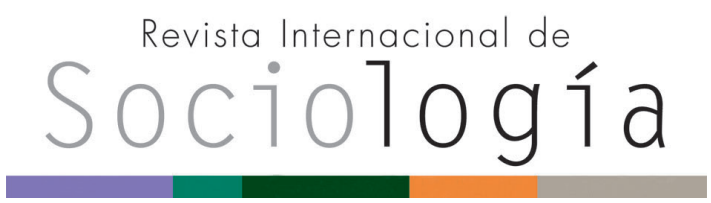

Revista Internacional de Sociología RIS vol. 75 (4), e078, octubre-diciembre, 2017, ISSN-L:0034-9712 doi: http://dx.doi.org/10.3989/ris.2017.75.4.17.04

\section{USOS, RESISTENCIAS Y ACEPTACIÓN DE TECNOLOGÍAS ENERGÉTICAS EMERGENTES EN EL HOGAR El caso de la política de recambio de estufas en Temuco, Chile}

\author{
Àlex Boso \\ Universidad de la Frontera, Chile \\ alex.boso@ufrontera.cl \\ ORCID iD: http://orcid.org/0000-0002-0313-8238 \\ TOMÁs ARIZTíA \\ Universidad Diego Portales, Chile \\ tomas.ariztia@udp.cl \\ ORCID iD: http://orcid.org/0000-0001-5806-3328 \\ FRANCISCA FonsECA \\ Universidad de la Frontera, Chile \\ francisca.fonseca@ufrontera.cl \\ ORCID iD: http://orcid.org/0000-0002-7663-7871
}

Cómo citar este artículo / Citation: Boso, A., T. Ariztía y F. Fonseca. 2017. "Usos, resistencias y aceptación de tecnologías energéticas emergentes en el hogar. El caso de la política de recambio de estufas en Temuco". Revista Internacional de Sociología, 75 (4): e078 doi: http://dx.doi. org/10.3989/ris.2017.75.4.17.04
USES, RESISTANCES AND

ACCEPTANCE OF EMERGENT

HOUSEHOLD ENERGY

TECHNOLOGIES

The case of the wood-burning stove exchange program in Temuco, Chile
Copyright: (C) 2017 CSIC. Este es un artículo de acceso abierto distribuido bajo los términos de la licencia Creative Commons Attribution (CC BY) España 3.0.

Recibido: 15/12/2016. Aceptado: 10/07/2017.

\section{Resumen}

En el marco de un creciente interés de la sociología del medioambiente y la energía por estudiar los procesos de transición hacia sistemas energéticos proambientales, este artículo presenta los principales hallazgos empíricos de un estudio de caso que aborda cualitativamente una política de recambio de estufas en la ciudad de Temuco, Chile. El artículo utiliza dos enfoques teóricos complementarios, la Multilevel Perspective (MLP) y la teoría de las prácticas sociales (TPS) para describir la existencia de mecanismos de bloqueo a los procesos de transición sostenible, tanto a nivel institucional como a nivel de las prácticas asociadas al uso de las estufas. El artículo se basa en 23 entrevistas semiestructuradas
\end{abstract}

\begin{abstract}
During recent years, there has been a growing sociological interest for studying transitions to pro-environmental energy systems. Against this backdrop, this article presents the main empirical findings of a case study that qualitatively addresses a policy of replacing stoves in the City of Temuco, Chile. The article uses two complementary theoretical approaches, the Multilevel perspective (MLP) and the Theory of Social Practices (TPS) to describe the existence of lock-in mechanisms for sustainable transition processes, both at institutional level and at the level of social practices through which heating is organized. The articles relies on 23 semistructured interviews with key stakeholders of an stove
\end{abstract}


a actores clave del proceso de recambio y 23 ejercicios de observación participante orientados a describir las prácticas de calefacción de los hogares de Temuco. En base a esta evidencia, el artículo plantea la necesidad de abordar las transiciones energéticas proambientales articulando enfoques teóricos que permitan dar cuenta de las distintas escalas y actores que constituyen este tipo de procesos. Este enfoque puede facilitar una mayor sensibilidad a las distintas dificultades inherentes a los procesos de transición energética.

\section{Palabras Clave}

Energía; Sociología de la Energía; Teoría de las Prácticas Sociales; Teoría Multinivel; Transiciones energéticas. replacement process and 23 participant observation exercises aimed at describing the heating practices of Temuco households. Based on this evidence, the paper reinforce the need to address different theoretical lenses in order to better account for the different scales and actors that constitute energy transitions. Such an approach can facilitate greater sensitivity to the difficulties that constitute pro environmental energy transition processes.

\section{KEYWORDS}

Energy; Energy Transitions; Multilevel Perspective; Social Practice Theory; Sociology of Energy.

\section{INTRODUCCIÓN}

En las últimas décadas, diversas ciudades del sur de Chile han sido afectadas por la contaminación atmosférica. Temuco y Padre Las Casas constituyen un caso paradigmático. Con una población de aproximadamente 340.000 habitantes, Temuco y Padre Las Casas están situadas en la mitad sur del país, a una altitud de 122 metros sobre el nivel del mar. Al igual que sucede en otras ciudades del sur, su clima es lluvioso y relativamente frío en invierno, cuando la temperatura mínima media alcanza los 3 grados. Según los datos del Ministerio de Medioambiente, aproximadamente el $81 \%$ del consumo de energía de los hogares del sur de Chile corresponde a la calefacción, siendo la leña el combustible más empleado (Ministerio del Medio Ambiente 2016). La situación de ambas ciudades, instaladas en un valle poco profundo, genera condiciones de inversión térmica en numerosos días del año y, por consiguiente, niveles altos de contaminación atmosférica. Solo en la primera mitad del 2016 se registraron 70 días con niveles de contaminación por encima de la norma (20 $\mu \mathrm{g} / \mathrm{m}^{3}$ ), de los cuales, 54 , llegaron a ser declarados como preemergencia o emergencia ambiental (Íbid.).

Los estudios epidemiológicos relacionan la contaminación atmosférica resultante de la quema de leña con la incidencia de numerosas enfermedades, que van desde afecciones leves, como dificultades al respirar o irritación en los ojos, hasta problemas de salud más graves, como el desarrollo de disritmia, infartos, bronquitis crónica o cáncer (Naeher et al. 2007; Hine et al. 2011). Así, en el humo producido por la quema de leña se encuentran diversos hidrocarburos aromáticos volátiles con propiedades carcinógenas como los isómeros de benceno, tolueno, etilbenceno y xileno (BTEX) o el material particulado fino $\left(\mathrm{PM}_{10}\right)$ y ultrafino $\left(\mathrm{PM}_{2.5}\right)$ (Bäfver et al. 2011; International Agency for Research on Cancer 2010). Por su lado, los problemas de salud derivados de la quema de leña no se distribuyen de manera homogénea en la población. Algunos estudios a nivel internacional indican que son los grupos vulnerables

los más afectados, quienes además, en numerosas ocasiones sufren situaciones de privación de los servicios de energía o pobreza energética (Boardman 2010; Ochoa y Graizbord 2016; Walker et al. 2016).

Alertados por la situación, el Gobierno central y las autoridades regionales han tratado de responder al problema. Temuco y Padre Las Casas fueron las primeras ciudades chilenas que implementaron sistemas de medición de emisiones de material particulado $\mathrm{PM}_{10}$ y $\mathrm{PM}_{2.5}$. A su vez, ambas comunas fueron pioneras en el desarrollo de políticas orientadas a reducir la contaminación ambiental, cuyo objetivo central es la transformación del sistema de tecnologías de calefacción para el hogar. En este contexto, surge el Plan de Descontaminación Atmosférica de Temuco y Padre las Casas, ideado desde el Ministerio del Medio Ambiente e implementado por los Seremis ${ }^{1}$ y autoridades locales. Este Plan cuenta con tres ámbitos de regulación: i) uso y mejoramiento de la calidad de la leña; ii) mejoramiento de la eficiencia térmica de la vivienda; iii) uso y mejoramiento de la calidad de los Artefactos, en el que se inserta el programa de recambio de calefactores.

Su objetivo es acelerar la tasa normal de sustitución, retirando artefactos a leña en uso (con alta emisión de partículas y baja eficiencia) e introduciendo calefactores que permitan lograr una mejora en la calidad del aire (con alta eficiencia de combustión y mejor eficacia, produciendo más calor a un menor costo). El Estado subsidia en un 100\% la compra del nuevo aparato de calefacción, el cual puede ser a pellet o parafina. Por medio de este programa, los beneficiarios pueden acceder a un nuevo calefactor siempre y cuando hagan entrega de su antiguo calefactor y/o cocina, el cual debe estar instalado y en uso en la vivienda. La meta de este programa es lograr 39.000 recambios de artefactos en las dos comunas para el año 2020 (Ministerio del Medio Ambiente, 2016). Al mes de noviembre del 2016, el proceso de recambio lleva un total de 1441 instalaciones distribuidas en dos etapas. Pese a que recientemente se está proyectando un nuevo sistema de postu- 
lación barrial para dinamizar el programa, los datos apuntan a que el proceso de transición energética en Temuco se encuentra obstaculizado por diversos mecanismos de bloqueo. El objetivo del artículo es examinar cómo operan dichos mecanismos, tanto en la escala institucional como en las prácticas sociales.

Hacer frente al problema de la contaminación atmosférica de las ciudades del sur de Chile implica transformar sus sistemas socio-técnicos. En el marco de los desafíos que impone el cambio climático, con una creciente demanda por eliminar las fuentes de carbón de la matriz energética, este proceso pasa por la adopción de fuentes y tecnologías basadas en energías renovables. El reto de descontaminar y, de forma concurrente, asegurar un abastecimiento de energía sostenible y asequible para la calefacción del hogar tiene importantes derivaciones. La transición de los sistemas de energía del sur urbano de Chile requiere la aceptación social de nuevas tecnologías y artefactos para calentar el hogar y, en paralelo, se precisa una modificación profunda de los patrones de demanda, los cuales están profundamente imbricados en la fábrica de la vida cotidiana de las familias que utilizan estufas a leña. Aunque la literatura especializada maneja múltiples definiciones de aceptación, para el propósito de este estudio seguiremos la empleada por Upham et al. (2015: 103), a saber: "Una respuesta favorable (incluida la actitud, intención, comportamiento y -cuando es adoptadauso) relacionada con una tecnología propuesta (o sistema socio-técnico) que es realizada por miembros de una unidad social dada (país, región, comunidad, ciudad, hogar u organización)". La aceptación suele ser vista como una de las dimensiones clave de las reacciones sociales hacia las tecnologías energéticas emergentes. Sin embargo, traspasando su dimensión psicosocial, la aceptación de tecnologías e infraestructuras emergentes es un elemento más en un proceso dinámico y complejo de transición sociotécnica, en el cual individuos y grupos de actores interactúan con tecnologías y desarrollos energéticos (Devine-Wright 2005; Oltra et al. 2012; Poumadère et al. 2015; Upham et al. 2015).

Por consiguiente, el estudio de dicho fenómeno debe abordarse con una mirada ecléctica que se centre en la dimensión sociológica de las políticas de intervención tecnológica en materia de energía y medioambiente. Concretamente, el artículo aborda dos enfoques teóricos complementarios, la Multilevel perspective (MPL) (Geels 2002, 2004, 2005) y la teoría de las prácticas sociales (TPS) (Shove et al. 2012) para examinar la política de sustitución de aparatos calefactores en el sur de Chile. Si bien ambas teorías han tenido un importante desarrollo en la literatura del mundo anglosajón, su adopción en otros contextos ha sido relativamente escaso. Así mismo, el estudio pretende realizar una contribución a la aplicación del enfoque MLP en contextos locales. Si bien diversos autores han remarcado el papel que pueden jugar las ciudades en los procesos de transición de sistemas de energía y aceptación de tecnologías emergentes, las contribuciones "clásicas" de la MLP no suelen ofrecer una visión a pequeña escala. Así, los estudios que parten del enfoque MLP para abordar el nivel local son escasos, 0 solo abordan lo local en relación al análisis de nichos (Mattes et al. 2015).

Aplicando el enfoque multinivel y el enfoque de la teoría de las prácticas sociales, este artículo analiza el papel de ciertos mecanismos de bloqueo que afectan los procesos de transición sostenible, tomando como caso de estudio el recambio de las "estufas a leña" en Temuco. El concepto de "bloqueo" (lockin) ha sido utilizado ampliamente para explicar la persistencia de sistemas tecnológicos basados en combustibles fósiles, a pesar de su conocida incidencia negativa en el cambio climático (Pierson 2000; Foxon 2002; Unruh 2002; Frantzeskaki y Loorbach 2010). Un bloqueo suele definirse como un mecanismo de amarre que, generalmente, se produce por una retroalimentación positiva o rendimientos crecientes vinculados a la adopción de una tecnología. Debido a la presencia de dichos mecanismos, las tecnologías establecidas en períodos tempranos tienen ventajas sobre innovaciones y desarrollos emergentes, no porque sean necesariamente mejores o más eficientes, sino porque han sido ampliamente difundidas y utilizadas. Los resultados de esta investigación muestran que es posible extender el concepto bloqueo a mecanismos que operan a escala de hogar y están imbricados en prácticas sociales. De tal forma, se sugiere que el diseño de las políticas para el control de la contaminación urbana deberá generar instituciones capaces de desbloquear mecanismos anclados a diferentes escalas. Las lecciones aprendidas en este estudio refuerzan la tesis promovida por otros autores, quienes afirman que los conceptos abordados desde una sola perspectiva teórica raramente nos proveen de conocimiento profundo y necesario acerca de las interacciones humano-energía, para poder orientar a los usuarios hacia comportamientos proambientales (Stern 2014; Upham et al. 2015).

El artículo se estructura como sigue. En la próxima sección se presentan las principales características de la perspectiva multinivel y de la teoría de las prácticas sociales, y su aplicación para el análisis de las políticas energéticas y medioambientales. Seguidamente, se describen brevemente los fundamentos metodológicos de la investigación. El corazón del artículo lo constituye la sección de resultados en la que se discuten en detalle cuatro mecanismos de bloqueo que pudieron ser identificados mediante las teorías de MLP y TPS. Por una parte, utilizando la literatura de MLP, discutimos dos mecanismos de corte institucional que dificultan este tipo de políticas 
de recambio, a saber, i) alta densidad institucional y problemas de coordinación; y ii) la existencia de asimetrías de poder. Complementariamente, utilizando la TPS, discutimos cómo la introducción de nuevas estufas se enfrenta a tensiones y resistencias que se dan a nivel de las prácticas de calefacción u otras adyacentes. En concreto, se analiza cómo las nuevas estufas contribuyen al cambio en la ecología de prácticas asociadas a la calefacción e implican la adopción de nuevas formas de mantenimiento y cuidado, que a su vez dificultan la propia adopción de estas nuevas tecnologías.

\section{MARCo TEÓRICO}

Existen dos tradiciones teóricas que pueden ser de gran utilidad para pensar, desde la sociología, sobre la aceptación y uso de tecnologías energéticas alternativas a las estufas de leña, en el contexto urbano del sur de Chile. Ambos enfoques, se caracterizan por proponer un acercamiento multidisciplinario a las transiciones tecnológicas en el ámbito de energía y por dar cuenta de la interrelación de aspectos tecnológicos, materiales y sociales.

Una primera tradición corresponde a lo que se ha denominado la teoría "multinivel" (MLP), vinculada a los estudios sobre transiciones energéticas. En los últimos años, las investigaciones sobre transiciones de sistemas de energía como procesos multinivel han proliferado en un área de investigación en la que confluyen disciplinas como la historia de la tecnología, la sociología y la economía evolutiva y los estudios de la ciencia, tecnología e innovación (Geels 2002, 2004, 2005; Loorbach y Rotmans 2006; Verbong y Geels 2007; Verbong y Geels, 2010). Toda transición conlleva una amplia serie de cambios de un régimen socio-técnico a otro, con el fin de establecer un nuevo sistema socio-técnico. Este enfoque, que consiste en analizar los cambios que se producen en el sistema de energía durante varias décadas, ha aportado información clave para entender cómo las tecnologías, regulaciones y actores interesados generan rutinas que orientan las decisiones de los usuarios hacia un comportamiento energético medioambientalmente insostenible (Geels 2002, 2004). Por otro lado, las teorías de las transiciones como procesos multinivel ayudan a comprender cómo las rutinas dominantes en el uso de energía pueden llegar a desestabilizarse, cómo se crean nuevas, o cómo pueden alinearse diferentes elementos de un sistema para desembocar en otro (Geels 2002, 2004, 2005).

El enfoque teórico del MLP describe las transiciones de los sistemas de energía como procesos en los que la evolución se produce por interacción a tres niveles: (i) régimen; (ii) paisaje; y (iii) nicho ${ }^{2}$ (Geels 2002, 2004). El régimen representa la configuración socio-técnica dominante, estabilizada por la interacción de elementos materiales existentes (tec- nologías, infraestructuras), grupos sociales y redes de actores, así como reglas o rutinas compartidas. Estos conjuntos de reglas son estables y orientan el comportamiento de los diferentes actores sobre cómo producir, regular o utilizar las tecnologías energéticas. Los regímenes socio-técnicos están integrados en los paisajes, que se definen como acontecimientos y tendencias exógenas que configuran la dinámica entre nichos y regímenes. Un nicho es un espacio, generalmente protegido de las presiones directas del mercado, en el que se desarrollan soluciones que de algún modo comprometen la lógica establecida por los regímenes. Los nichos provienen de arreglos socio-técnicos a pequeña escala; se constituyen con unos pocos actores, con interrelaciones escasas, pues en realidad hacen las veces de bancos de pruebas de diferentes configuraciones alternativas de elementos materiales, actores y reglas. Según las teorías del MLP los cambios ocurren cuando el paisaje evoluciona y ejerce presión sobre un régimen, en el que se intensifican sus tensiones internas. Dicha situación, genera una ventana de oportunidad para soluciones tecnológicas que emergen y maduran en los nichos. Los cambios en los sistemas socio-técnicos suelen ser incrementales. Es decir, el régimen incorpora de manera paulatina algunos elementos de nichos emergentes sin modificar en exceso su lógica fundamental.

La teoría de las prácticas sociales (TPS) es clave para entender cómo las transiciones energéticas se ven frenadas por mecanismos de bloqueo que operan en los hogares. En lo que sigue describimos sucintamente esta teoría, en particular su adaptación al estudio del consumo sostenible y al uso de energía desarrollado por Elizabeth Shove y colegas, el cual ha sido utilizado para el estudio de procesos de transición tecnológica y el potenciamiento de formas de consumo sustentable y de estilos de vida bajos en carbón (Shove 2003; Shove et al., 2012; Shove y Walker 2014; Walker 2014; Warde 2012; Strengers 2012; Strengers y Maller 2015; Welch y Warde 2015; Crivits y Paredis 2013; Ariztía 2017).

A diferencia de la teoría MLP, la cual concentra su análisis en la dimensión macro y meso de los procesos de cambio tecnológico, la TPS pone su atención preferentemente en la dinámica micro-sociológica, sobre la cual se estructuran las dinámicas de uso de energía. La TPS propone entender las distintas formas de consumo -incluyendo el uso de energíacomo resultado de la dinámica de las prácticas que organizan la vida cotidiana de los usuarios. Siguiendo a Schatzki (1996), Shove propone definir a las prácticas como una forma rutinizada de conducta compuesta por un "nexo de formas de decir y de hacer que tiene cierta dispersión espacial y temporal" (1996). Desde aquí, es posible distinguir tres componentes principales de toda práctica: el conocimiento práctico (el "saber hacer"), las materialidades (los 
objetos materiales y recursos que constituyen la práctica) y el sentido (el conjunto de significaciones y valoraciones que constituyen cada práctica) (Shove, Pantzar y Watson 2012, cap. 1). Cada práctica depende para su existencia de la interrelación activa de estos tres elementos. En el caso de la práctica de cocinar, por tomar un ejemplo, no solamente se necesita "saber cocinar", sino también contar con los distintos objetos necesarios (instrumentos, recursos) y disponer de las motivaciones y las valoraciones adecuadas para hacerlo (querer cocinar para la familia o valorar el cocinar como una actividad relevante).

Una de las fortalezas centrales de la TPS es que su principal unidad de análisis, las prácticas, no son resorte únicamente de la intencionalidad de los actores, sino que dependen de distintos elementos que la constituyen y que trascienden la intencionalidad. Dicho de otro modo, la práctica como unidad no se refiere únicamente al ámbito de la intencionalidad individual, sino también a un conjunto de materialidades y significados que se movilizan en ella y anteceden a su ejecución individual. Por ejemplo, la práctica de prender una estufa puede ser estudiada como una unidad autónoma, con una historia y trayectoria que van más allá de las instancias particulares en que sujetos ejecutan la práctica. Esto implicaría estudiar la trayectoria de las tecnologías asociadas a prender estufas, la forma en que se ha producido y diseminado el conocimiento práctico sobre las estufas y las distintas significaciones de su uso.

Desde la TPS la vida social es entendida como un entramado de prácticas relacionadas y cuya dinámica da forma a la vida cotidiana estructurando los patrones de demanda. En relación a los patrones de consumo y adopción de tecnologías asociadas al uso de energía, el foco de la teoría de las prácticas se ha centrado en comprender cómo distintas configuraciones de práctica implican distintos niveles de uso de energía a nivel cotidiano (Shove y Walker 2014). Como señalan distintos autores, esta aproximación tiene la fortaleza de visibilizar el aspecto rutinario y convencional de la vida social, que muchas veces explica cambios en los patrones de consumo y el éxito (o no) de transiciones hacia regímenes de consumo sustentables (Shove 2010; McMeekin y Southerton 2012). Este planteamiento se vincula a una larga tradición de estudios empíricos etnográficos en las prácticas de uso energético con atención a las particularidades y complejidades en que ellos están situados (Wilhite et al.1996).

La TPS permite reflexionar acerca de qué configuraciones de prácticas -entramado de saberes, tecnologías y significados- facilitan una transición energética hacia regímenes de bajo consumo en carbón. En este contexto, las distintas tecnologías y los procesos de cambio tecnológico son abordados en relación con su capacidad para afectar a la dinámica de las prácticas cotidianas preexistentes. Algunos procesos de transformación tecnológica pueden, por ejemplo, volver inviable el desarrollo de ciertas prácticas altamente valoradas, por tanto, su incorporación se vuelve dificultosa. De la misma forma, estos procesos pueden requerir niveles de experticia práctica asociada a su incorporación, dificultándose su masificación. Esta línea de análisis, es afín a la propuesta por los estudios de ciencia y tecnología; en particular, los estudios de adopción de tecnologías y su foco en las distintas posibilidades de utilización que presentan los objetos tecnológicos, la forma en que permiten distintos tipos de interacción y modificación de sus aptitudes (Laet y Mol 2000).

Recientemente algunos autores han buscado vincular la TPS con los enfoques MLP asumiendo que ambas perspectivas proponen formas relativamente novedosas de pensar los procesos de cambio tecnológico y de transición hacia regímenes sustentables en forma multidimensional. En este marco se ha propuesto que la teoría de las prácticas logra complementar adecuadamente a nivel micro la explicación de procesos que son tratados por MLP solo a nivel meso o macro societal. A su vez, la teoría MLP ofrecería a la TPS una posibilidad de apartar el foco, en ocasiones, excesivamente centrado en el consumo final, incorporando así aspectos sistémicos en sus estudios (McMeekin y Southerton 2012; Crivits y Paredis 2013).

Siguiendo esta línea, proponemos que tanto el enfoque del MLP como la TPS puedan ser de utilidad para caracterizar el caso de Temuco, Chile, como un proceso de transición hacia un nuevo sistema de energía. Sin embargo, a partir de las lecciones extraídas del análisis de este caso, sostenemos que la perspectiva teórica del MLP puede mejorar su capacidad explicativa de las transiciones de los sistemas de energía, en la medida en que los estudios que orienta complementen adecuadamente la descripción y análisis de los procesos de aceptación de tecnologías energéticas emergentes con foco en las prácticas concretas asociadas a la utilización de estas tecnologías. Para desarrollar estos aportes, nos centramos en la capacidad de ambas teorías para explicar la existencia de mecanismos de bloqueo que dificultan el despliegue de transiciones energéticas que operan como sistemas complejos, y que involucran una transformación radical de las prácticas sociales que se dan cotidianamente en los hogares.

Uno de los argumentos centrales de este trabajo es que la persistencia de los sistemas socio-técnicos existentes puede ser explicada por el uso de conceptos más específicos que los de nicho, régimen socio-técnico y paisaje -enfoque MLP-. Desde esta perspectiva teórica se suele argüir que los diferentes elementos de un sistema complejo -las dimensiones materiales, organizativas y conceptuales del sistema- están alineadas entre sí dotándolo de cierto equilibrio y permanencia (Kemp et al. 1998; Geels, 2004; Sandén y Hillman 2011;). Así, el sistema socio- 
técnico existente suele tener una influencia estabilizadora en la dinámica de la innovación y obstaculiza la introducción de tecnologías que provoquen un cambio radical de trayectoria. Sin embargo, la perspectiva de la MLP describe el bloqueo de una manera bastante generalizadora, con pocas descripciones de los procesos específicos a través de los que se manifiestan (Kiltkou et al. 2015). De hecho, tal como han constatado recientemente algunos autores, MLP carece de una teoría adecuada que describa la dinámica de los procesos de aceptación, adopción y consumo final (McMeekin y Southerton 2012). Al analizar el caso del recambio de las estufas de leña en Temuco, pretendemos mostrar que una distinción más precisa de la forma de cómo operan los mecanismos de bloqueo tecnológico puede mejorar la comprensión de la persistencia de regímenes socio-técnicos dominantes y de las dificultades de los nichos emergentes para crecer y consolidarse. En este sentido, tal como han planteado recientemente algunos autores (Crivits y Paredis 2013), la TPS puede ofrecer un muy necesario complemento para especificar algunos de estos mecanismos a nivel de usuarios.

\section{Metodología}

El artículo se basa en información aportada por participantes implicados en el proceso de aceptación de tecnologías energéticas alternativas a la estufa de leña. En concreto, las 2 fuentes principales de datos de esta investigación provienen de: (i) la aplicación de 23 entrevistas semiestructuradas con una muestra de actores clave (técnicos, representantes de la industria local, representantes de los gobiernos y las secretarías ministeriales de la región y usuarios finales) en las comunas de Temuco y Padre Las Casas; y (ii) la realización de 23 observaciones participantes complementadas con entrevistas semiestructuradas cuyo principal foco fue dar cuenta de las prácticas de calefacción de los hogares, en 10 de los cuales se realizó el recambio a un nuevo calefactor a pellet o a parafina. En cada hogar se realizaron como mínimo dos visitas, a fin de desarrollar observaciones directas y entrevistas en profundidad, que nos permitieran describir los aspectos generales de la práctica de calefacción. Durante estas visitas, se discutieron distintos aspectos relativos al proceso de recambio y a la utilización de las nuevas estufas. Esto fue complementado durante 2016 por un seguimiento a todo el proceso de recambio de estufas a leña por pellet y parafina, comenzando con la postulación, visita de confirmación de antecedentes presentados para ser objeto de recambio, selección de los hogares, capacitación de las empresas que recambian los artefactos y el recambio de estufas.

El trabajo de campo se realizó durante los meses de junio a octubre de 2016. Para responder a las preguntas de investigación, principalmente, se analizó información cualitativa de dichas fuentes, con ciertos datos cuantitativos (por ejemplo, número de estufas instaladas en las políticas de recambio o número de sumarios realizados en los períodos de restricción por contaminación). Los participantes se seleccionaron mediante un muestreo deliberado para asegurar que todas las visiones y partes implicadas en el proceso de transición fueran incluidas en la investigación. Las entrevistas y la observación participante en los hogares fueron aplicadas con la ayuda de un guion predeterminado con preguntas orientadas a dar cuenta de los distintos aspectos de las prácticas, así como de los mecanismos bloqueo que, a diferentes escalas, podrían estar bloqueando el proceso de transición.

Las entrevistas y las observaciones participantes en los hogares fueron grabadas, transcritas y, a continuación, se analizaron a partir de un análisis temático (Boyatzis 1998; Krueger y Casey 2000; Braun y Clarke 2006; Fereday y Muir-Cochrane 2006). El análisis se realizó utilizando Atlas.ti. Este análisis permitió la generación inductiva y deductiva de códigos y la identificación de extractos pertenecientes a cada categoría temática. El análisis se ha centrado en estudiar los resultados obtenidos en las observaciones participantes y en las entrevistas semiestructuradas.

\section{Resultados}

A continuación se presentan los principales resultados del estudio. En primer lugar, se utiliza la teoría MLP para describir el caso e identificar dos mecanismos de bloqueo institucionales. En segundo lugar, mediante el enfoque de la TPS se discuten dos bloqueos que operan al nivel de las prácticas de uso de energía en el hogar.

\section{Teoría MLP y mecanismos de bloqueo institucionales}

Los artefactos a leña son la pieza fundamental del régimen socio-técnico de Temuco y Padre Las Casas. El consumo de leña para calefacción se aplica en el $90 \%$ de los hogares de ambas comunas (Ministerio de Desarrollo Social 2013). Existen una serie de actores y grupos de interés que regulan y construyen las rutinas que definen el régimen socio-técnico actual. A nivel formal, los Seremi (Secretarías Regionales Ministeriales) de Energía, Salud, Medioambiente y Vivienda constituyen piezas claves del régimen, pues, a través de los Planes de Descontaminación $y$ otros instrumentos establecen una serie medidas para el control de la contaminación atmosférica, combinando estrategias de regulación (mediante un sistema de sanciones e incentivos) y de provisión de información. A su vez, dada la ausencia de un marco legal nacional que regule la leña como combustible y los fuertes vínculos entre el medio rural y urbano que caracterizan a la región, la cadena que va desde la producción al consumo de leña se desenvuelve en un mercado relativamente no formalizado. 
A nivel de paisaje, dos elementos presionan al régimen actual. En primer lugar, la contaminación atmosférica (por emisión de la propia combustión de leña) genera problemas de salud pública y, por consiguiente, una reacción de las autoridades regionales que tratan de implementar políticas para favorecer una transición energética. Por otro lado, en la última década la población en las comunas de Temuco y Padre Las Casas ha aumentado un $16 \%$, convirtiéndose en el principal polo de atracción de la migración rural de la propia región (INE 2016). Este cambio demográfico constituye una fuente de presión para el régimen socio-técnico, pues el aumento de demanda de leña empeora la calidad del aire en la ciudad.

La proliferación de nichos tecnológicos emergentes es consecuencia de la presión que el paisaje ejerce sobre el régimen. El crecimiento de estos nichos es, en parte, estimulado y protegido por una serie de subsidios públicos, cuyo máximo exponente es la política de recambio de estufas de leña. Existen diversos nichos tecnológicos emergentes en Temuco, aunque tres de ellos parecen ser predominantes: (i) las estufas a pellet, (ii) las estufas a parafina; y (iii) la calefacción distrital. Esta última es presentada como una apuesta de futuro por parte de las autoridades políticas, aunque tiene una baja penetración en la matriz energética de la ciudad. Por esta razón, en esta sección nos centraremos en describir dos mecanismos de bloqueo que a escala institucional bloquean la penetración de las estufas a pellets y parafina.

\section{Alta densidad institucional y problemas de coordinación}

El entramado de instituciones que regulan el régimen socio-técnico y la transición energética de Temuco es relativamente elevado en cuanto a su número, y complejo en lo que atañe a su coordinación. Como es sabido, los efectos de coordinación ocurren cuando los beneficios que un individuo recibe de una actividad particular se incrementan cuando otros adoptan la misma opción. Dichos efectos son particularmente críticos cuando una tecnología debe ser compatible con una infraestructura vinculada, como en el caso de las estufas a pellets y el aislamiento térmico de las viviendas. A pesar de la voluntad de los responsables de las políticas, en ocasiones, la alta densidad de las instituciones provoca un bloqueo en la transición por problemas de coordinación. En el caso de Temuco, ciertas políticas son interdependientes, se supedita el éxito de una al de la otra. Valga como ejemplo ilustrativo la relación entre la política de recambio, las políticas de reacondicionamiento térmico de la vivienda y la política de restricción de uso de estufas a leña.

En Temuco, el coste de la estufa a pellets, su instalación y mantenimiento, dificulta su aceptación en- tre los usuarios. A sabiendas de este inconveniente, las autoridades impulsaron la política de recambio de estufa a leña por otra tecnología más eficiente, subvencionado completamente el recambio. No obstante, en alguna de sus fases de aplicación, se incorporó como prerrequisito fundamental para puntuar en la postulación un adecuado aislamiento térmico de la vivienda beneficiaria. Según la visión de los responsables de la política, técnicamente no tiene sentido sustituir si no tienes aislada la casa, pues el coste de calentar la vivienda se incrementa:

\begin{abstract}
"La gran barrera o punto crítico ahí es que en Chile las casas no están bien aisladas. Cualquier salto de leña a otra tecnología se hace imposible si tú no tienes... Es decir, se hace muy costosa, si tú no tienes la casa aislada. Si no tienes cierta eficiencia energética, un mejoramiento técnico se hace crítico. El costo de calefacción se te va a ir a las nubes, se dispara. Hay que tener en cuenta que en estos equipos inteligentes como pellet o parafina su operación se enlaza a demanda energética de la vivienda. Si no tienes la casa aislada la vivienda está demandando calor constantemente, y la estufa no deja de trabajar". (Funcionario Seremi MMA).
\end{abstract}

La dependencia entre la estufa a pellets y la calidad del aislamiento del hogar hace necesario un esfuerzo de cooperación entre los responsables de las políticas de recambio y de vivienda. Actualmente, las condiciones para postular a uno u otro incentivo ejercen de facto como mecanismo bloqueo. El aislamiento térmico es un déficit que afecta a gran parte de las viviendas de la región. Por un lado, existen barrios que se construyeron décadas atrás como asentamientos provisionales para recibir la llegada de personas desplazadas por un terremoto (sector Millaray, en Javiera Carrera). Esas viviendas fueron diseñadas y construidas como solución coyuntural, pero, finalmente, se asentaron como barrios. Por otro lado, según la percepción de los expertos entrevistados, la actual legislación chilena en materia de aislamiento de vivienda es demasiado laxa. Así, pese a cumplir con la normativa, las casas de nueva construcción presentan déficits en cuanto a su aislamiento, si se considera el clima lluvioso y frío de la región. Además, a pesar de la existencia de subsidios para reacondicionamiento térmico de viviendas antiguas, los criterios de elegibilidad (por ejemplo, ser propietario de la vivienda con la documentación al día, incluido el registro de todas las reformas realizadas) y el copago exigido hacen que los estratos de población más vulnerables raramente salgan beneficiados, minimizándose así sus opciones de optar al recambio. En definitiva, los problemas de coordinación institucional hacen que la adopción de estufas a pellets en los hogares más vulnerables sea escasa, pese a la presencia de subsidios estatales.

La política de restricción de uso de calefacción a leña, también está vinculada con el recambio, siendo un acicate para que tecnologías energéticas emer- 
gentes ganen cotas de mercado a las estufas a leña. En la medida en que los usuarios ven mermada su capacidad de utilizar las estufas a leña en períodos de preemergencia o emergencia, es de esperar que se planteen su recambio. Así, la mayoría de los expertos entrevistados consideran que las restricciones son clave para promover la aceptación social de nuevas tecnologías y de combustibles alternativos a la leña:

\footnotetext{
"Es súper relevante tener restricción, por ejemplo hoy tenemos un pronóstico de que no se pueden usar las estufas después de las seis de la tarde, ha jugado un papel importante como impulsora, como un motor que lleva a que la gente que de mayores ingresos sin se cambien a otros combustibles". (Técnico Seremi MMA).
}

Sin embargo, las políticas de restricción están temporalmente descoordinadas con el programa de recambio (pues las primeras se aplican solo en invierno) y se han mostrado limitadas en su aplicación. En Temuco las autoridades sanitarias prohíben el uso de estufas de leña en episodios de preemergencia y emergencia sanitaria a partir de las 6 de la tarde y hasta las 6 de la mañana del día siguiente. No obstante, la norma se aplica por polígonos, para proteger a los sectores poblacionales vulnerables, quienes difícilmente tienen acceso a otro tipo de calefacción por motivos económicos. Solo el $50 \%$ del área residencial está sujeta a la restricción de uso de artefactos a leña. La normativa tampoco aplica a aquellos hogares en los que residen adultos mayores o personas enfermas. Por otro lado, no todas las visitas realizadas a domicilios que están violando la norma acaban en sanción. Los responsables de políticas de restricción entrevistados afirman que desde abril a agosto de 2016 (momento en el que se realizó la entrevista) se habían realizado 2422 fiscalizaciones, pero menos de la mitad (1036) causaron sumario.

El "patrullaje de chimeneas" (fiscalización de viviendas) se basa en la asignación de inspectores en 10 micropolígonos en los que rige la restricción. Los inspectores visitan aquellos hogares que, según su observación, están emitiendo humos visibles. Los expertos entrevistados explican que los usuarios tratan de evitar las multas aludiendo situaciones específicas. En general, piensan que ciertos alegatos están justificados, como es el caso de viviendas en las que se hallan personas enfermas o cuyas condiciones económicas son extremadamente precarias. No obstante, según el relato de los inspectores entrevistados, en determinadas ocasiones existe una clara voluntad de no cumplir la norma. Por ejemplo, algunos fiscalizadores comentan que las personas dejan sacos de leña seca en la sala de estar para declarar que esa es la que utilizan (cuando en realidad queman leña húmeda, que genera más emisiones) o, antes de recibir a los inspectores, abren el tiraje de la chimenea y echan un tronco al fuego, para alegar que justo acaban de cargar. En otras palabras, según la experiencia de los entrevistados los usuarios buscan estrategias para seguir empleando sus artefactos a leña, a pesar de las restricciones y del peligro de recibir sanciones.

En definitiva, las restricciones al uso de artefactos a leña tienen un éxito parcial y efectos limitados: no afectan a todos los barrios de la ciudad; no se sancionan todos los hogares que están incumpliendo la norma; y, ciertos individuos han encontrado estrategias que les permiten mantener comportamientos que no son proambientales (quemar leña húmeda o cerrar el tiraje de la estufa, incluso en periodos de restricción). El éxito del programa de recambio se ve también influenciado por este motivo. Si los usuarios no se sienten presionados por la política de restricción, la aceptación y posterior adopción de otras tecnologías menos contaminantes suele pasar a un segundo plano. Es decir, debido a la complementariedad existente entre ambas políticas, la transición energética se ve frenada por un bloqueo generado por la ausencia de coordinación institucional.

\section{Asimetrías de poder}

Las autoridades locales perciben que las estufas a pellets son una de las mejores alternativas tecnológicas para llevar a cabo la transición del sistema de energía de Temuco. Por ese motivo, las relaciones de cooperación entre las instituciones gubernamentales y la industria del pellet son fluidas. Durante la última década se ha fraguado un nicho tecnológico emergente capaz de disputar ciertas cuotas de mercado a las estufas a leña. El análisis de los datos muestra que la preferencia de los técnicos y representantes de la administración pública por el pellet sobre otras tecnologías emergentes está ligado a la promoción de la biomasa. Los expertos entrevistados opinan que es un "combustible renovable propio de la región". La industria de la leña (y de los artefactos relacionados con su uso) es uno de los sectores con mayor peso económico en sur de Chile. Por consiguiente, el recambio a un combustible como el pellet es bien valorado, dado que podría continuar generando beneficios económicos a la región. El grueso de los entrevistados piensa que el recambio masivo a otros combustibles, como la parafina o el gas natural, conllevaría una situación de dependencia energética y una mayor volatilidad de precios.

\footnotetext{
"Hay cierto giño al pellet. Básicamente, porque es una estrategia de desarrollo regional, de acá de la región de La Araucanía, respecto a combustibles y energías renovables y, en especial, a la biomasa. Entonces, en función de eso, nosotros impulsamos en el 2013 una campaña pública con respecto al pellet, hagamos difusión del pellet". (Técnico Seremi MMA).

[Respecto a los distribuidores de estufas a pellet] "Somos bien conocidos de ellos. Tenemos una relación bastante fluida en términos de información y ellos están siempre invitándonos a presentaciones y actividades. De hecho, ayer andábamos en un taller". (Técnico Seremi MMA).
} 
Sin embargo, a pesar de que el nicho tecnológico de la estufa a pellets se encuentra protegido por la administración local, la transición energética está frenada por mecanismos bloqueantes enraizados en asimetrías de poder. Aunque el recambio a estufa a pellets es percibido por los expertos como una buena solución tecnológica para una transición rápida y sostenible, existen actores que se verían perjudicados en el nuevo escenario. Los productores y distribuidores de tecnologías o combustibles alternativos al pellet, entre los que, lógicamente, se incluyen actores vinculados a la producción, venta y distribución de la leña aparecen como claros perdedores de la transición. Por ese motivo, en la medida de sus posibilidades, dichos actores movilizan recursos de poder para frenar los procesos de adopción de estufas a pellets impulsados por la administración.

\begin{abstract}
"Mira, nosotros hemos impulsado el pellet, derechamente sobre otros combustibles. Eso nos ha costado una visión negativa, especialmente, de parte de la industria de gas, pero también de otros sectores... Nos tiran para abajo todas las licitaciones. Cuando hemos comprado estufas a pellets, ellos han parado licitaciones, nos han impugnado la compra, diciendo: ¿Por qué el Estado compra pellet y no gas? ¿Por qué ese direccionamiento a ese combustible que no está normado". (Técnico Seremi MMA).
\end{abstract}

Los mecanismos de bloqueo basados en asimetrías de poder operan también a un segundo nivel. En ciertos bloqueos el poder no es impuesto de manera abierta, sino que se refleja en un conjunto de reacciones anticipadas. En general, la información analizada revela cierta ambivalencia en el discurso de la administración y los responsables políticos respecto a la leña. Por un lado, hay consciencia de los problemas de salud pública provocados por la leña y se han desarrollado una serie de políticas regulatorias y de provisión de información para manejar este problema ambiental. Pero, paradójicamente, en el discurso de los responsables, los expertos y los documentos oficiales se afirma que "la leña es un combustible moderno y limpio" o que la leña "puede y debe recuperar su prestigio" (Ministerio de Energía 2015). En definitiva, se observa que los responsables políticos perciben el mercado de la leña como un sector económico vigoroso en la región. Por consiguiente, en el diseño de políticas no se suelen plantear cambios radicales, ni se suele buscar activamente incentivos para una aceleración de la transición, pues se percibe que tales acciones generarían rechazo social.

\section{Teoría de las prácticas sociales y mecanismos de bloqueo: reconfigurando la práctica de calentar}

La perspectiva de las TPS agrega una mirada al programa de recambio de estufas examinado y, por tanto, permite identificar otro tipo de mecanismos en juego. Desde este enfoque, es posible observar cómo la incorporación de nuevas estufas redefine un entramando de prácticas que constituyen la vida doméstica. Para la TPS, los artefactos y otras formas de materialidad juegan un papel determinante en la organización de las prácticas cotidianas. Por tanto, la política de incentivo a la substitución de las estufas a leña implica una reconfiguración de las prácticas existentes. La nueva tecnología, ya sea esta estufa a pellets o parafina tensiona muchas de las prácticas de la vida cotidiana. En lo que sigue, se discuten dos tipos de tensiones que, siguiendo el argumento general del artículo, cabe identificar como mecanismos de bloqueo a nivel de prácticas en el hogar. A pesar de su relevancia, en este artículo hemos dejado deliberadamente fuera los elementos relativos a favorecer la sustitución de las estufas, para centrarnos en los mecanismos que la dificultan. Concretamente, se describen dos mecanismos: i) los efectos del cambio de estufa sobre la ecología de prácticas que constituye la vida doméstica, en particular, las tensiones que incorpora; y ii) la relación entre instalación de nuevas estufas y el despliegue, para su mantenimiento y cuidado, de nuevas prácticas y tecnologías.

\section{Cambio en la ecología de prácticas}

Desde la TPS se observa cómo la introducción de las nuevas estufas a pellet entraña un importante proceso de reconfiguración de las prácticas de calefacción y otras prácticas adyacentes en el hogar. Este proceso de cambio opera como un mecanismo de bloqueo que dificulta la adopción de tecnologías emergentes.

En los hogares del sur de Chile las cocinas y las estufas de combustión a leña son los artefactos mayormente empleados y de más prolongada existencia. Precisamente, constituyen objeto de la política de recambio por ser las principales fuentes de contaminación atmosférica y no por su eficiencia energética. Las estufas a leña y particularmente las cocinas, son artefactos amarrados a un repertorio de prácticas de calefacción tradicional, que se reproduce familiarmente entre generaciones. Calentar la casa está vinculado a un repertorio amplio de prácticas asociadas como cocinar, el lavado y secado de ropa y la sociabilidad en torno al fuego. Por tanto, cualquier modificación en estos aparatos reconfigura no solo la práctica de calentar la casa, sino también las prácticas adyacentes.

Un aspecto fundamental de esta reconfiguración es el conocimiento práctico asociado al manejo de las estufas a leña, que es sustituido por saberes de otro tipo. Por ejemplo, las estufas y cocinas tradicionales requieren manejar un notable conjunto de competencias relativas al combustible, uso y mantenimiento. Un ámbito relevante de competencias 
asociadas a estas prácticas implica la relación con el combustible que se utiliza y la forma de conseguirlo y prepararlo. En términos de las estufas tradicionales, los usuarios describen un conocimiento histórico del manejo de combustible que va desde la elección del tipo de leña para cada tipo de estufa, cortar/trozar, almacenar, hasta mantener y limpiar las estufas en las distintas épocas del año y en función del uso. Una vez que se ha comprado la leña, y almacenado para que no se humedezca, esta debe ser trozada en un tamaño más pequeño si se quiere usar en la cocina. Cuando se prende la cocina ${ }^{3}$ hay que saber manejar el fuego abriendo y cerrando los "tirajes" y se requiere una manutención rutinaria de las cenizas, que suele ser parte de las prácticas de aseo del hogar. Con la incorporación de las nuevas estufas, muchos de estos saberes prácticos se vuelven irrelevantes. Por ejemplo, se observa que un tema central en la respuesta de los beneficiarios tiene relación con la ausencia de competencias adecuadas para el manejo de las nuevas estufas. La mayoría dependen de sistemas de programación según grados y horarios, que difieren de las formas tradicionales de gestión y cuidado del fuego. Por ejemplo, en hogares con adultos mayores hay temor de estropear o desconfigurar el artefacto que dejaron programado los técnicos. Solo se utilizan las funciones básicas; encendido, apagado y el termómetro que especifica los grados de temperatura a alcanzar.

"La nueva estufa, no la programo, no lo necesito. En la mañana tipo diez la encendimos, se calienta rápido el ambiente, luego la apago antes de irnos a la cama, nos acostamos temprano... Tampoco sé cómo programarla y prefiero no hacerlo para no estropearla. Pero si tuviera que hacerlo tendré que pedirle a alguien que me explique, porque no recuerdo cómo fue que me lo explicó el técnico. Debe ser la edad".

(Señora S, agosto 2016).

En cuanto objeto, los artefactos a leña no solo posibilitan la calefacción sino una ecología de prácticas cercanas. En este sentido, las opciones materiales de la estufa a leña son centrales para asociarla con otras prácticas. Así, son objetos centrales en términos de la producción de agua caliente (a través de serpentín) y el lavado de ropa, la práctica de cocinar y la generación de instancias de sociabilidad. En algunos casos, dicha centralidad depende de procesos de adaptación técnica que faciliten las prácticas.

\footnotetext{
“...mi marido ha construido un sistema para secar la ropa, igual al que venden pero mucho más firme. Se pone en el cañón y en unas horas está seca. Si hay que tener cuidado de que no se quemen, porque si tocan el cañón incluso se puede provocar un incendio. También cocinamos en la estufa, la comida queda más rica, se cocina más lento, igual hervimos el agua, así tenemos siempre agua caliente..." (Señora M, septiembre 2016)
}

Las nuevas estufas redefinen el marco de las prácticas que se pueden beneficiar de ellas, reconfigurando un conjunto importante de prácticas adyacentes a la calefacción, como son el secado de ropa o la producción de agua caliente. Por ejemplo, los nuevos aparatos, sobre todo los aparatos a parafina, hacen difícil poder secar ropa, el tipo de calor que esta estufa emana no permite el secado de forma equivalente. Al desaparecer la práctica tradicional de secado en torno al cañón se generan también nuevos gastos e inversiones.

“....al no tener la cocina a leña se pierde el poder secar la ropa. Por eso tuvimos que comprar una secadora, antes la secábamos en el cañón. Es un gasto más, pero no se nota tanto..." (Señora X, septiembre 2016).

La sociabilidad familiar se ve reconfigurada por la incorporación de las nuevas estufas. La estufa a leña, particularmente la cocina, articula un espacio de vida en común en torno al aparato. Las alusiones a conceptos como "reunirse en familia", "el calor de hogar", "dar sabor a las comidas" y "recuerdos de tiempos pasados", son recurrentes en gran parte de los relatos que se han recopilado. Esto mismo genera dificultades en términos de adopción de las nuevas tecnologías, en parte porque problematiza un aspecto fundamental del hogar como la sociabilidad.

“...cuesta acostumbrarse porque el solo hecho de mirar la estufa, ya la forma es diferente. Psicológicamente ya me produce un choque porque la estufa a leña es algo, donde nos sentamos alrededor a tomar mate a hacer la vida social familiar en la casa. En cambio, con esta estufa a parafina no es igual, es como un mueble más...". (Señora "V3", octubre 2016).

Los datos describen una tensión asociada a la incorporación de nuevas tecnologías: la forma en que estos nuevos aparatos reconstituyen y vuelven problemáticas algunas prácticas existentes. Tal como ha sido discutida por la literatura de la TPS esta tensión hace referencia al hecho de que calentar la casa coexiste sincrónicamente con otras prácticas que conforman la vida doméstica (Shove et al. 2012).

\section{Mantenimiento y cuidado}

Existe un cuarto bloqueo relacionado con el conjunto de prácticas asociadas a la mantención y cuidado de las estufas. Las nuevas estufas incorporan un repertorio de prácticas de mantenimiento y operación distintas a las prácticas de cuidado de las estufas a leña tradicionales.

La mantención y el cuidado de los artefactos a leña conllevan un conjunto de prácticas cotidianas que están imbricadas en las rutinas familiares diarias. Es necesario limpiar las estufas todos los días antes de usarlas, retirar la ceniza y los restos de leña que quedan acumulados. También hay que limpiar la base de la cocina donde se ponen las ollas o cazuelas, empleando materiales que no rallen la superficie. 
La limpieza del cañón tiene cierta complejidad, pues se debe subir al techo y raspar desde allí con una herramienta con puntas de metal para desprender los restos de hollín y cenizas adheridos al tubo. De no hacerlo, se corre el peligro de incendio.

Las nuevas estufas son objetos "más delicados". Involucran prácticas de mantención y cuidado que, en la mayoría de ocasiones, no pueden ser desarrolladas por los usuarios. Así, se generan nuevas prácticas que están vinculadas a sistemas de mantención expertos. Tanto las estufas a pellets como a parafina necesitan de una revisión anual, en la que se desarma la estufa y se limpia, proceso que es llevado a cabo por un servicio técnico especializado. Si la revisión no es realizada por un servicio técnico autorizado por la marca de la estufa, la empresa no se hace responsable de posibles fallas de los aparatos. Además, el conocimiento técnico para la mantención y uso de los nuevos aparatos se transmite a través de manuales de instrucciones y otros documentos formales, que son ajenos al repertorio habitual de práctica de cuidado que realizan los usuarios. La aparición de estas prácticas, puede interpretarse como una nueva forma de "rigidez" que disminuye el involucramiento de los usuarios. En las entrevistas realizadas, se observa temor a estropear los aparatos y una dificultad para poder lidiar con los requerimientos de su mantención y cuidado.

"Si he necesitado ayuda. He leído el manual y me ha servido otras veces. Una vez tuve que llamar al técnico. ¿Por qué fue? No me acuerdo...Lo llame, me dio las indicaciones... Yo le decía que no funcionaba y al final era que yo no había cerrado bien la estufa".

(Señora X, septiembre 2016).

Esta distancia resulta evidente durante el proceso de instalación, cuando se enseñan una serie de acciones para poder utilizar y mantener operativa la estufa, que son relativamente ajenas a los usuarios. Con las nuevas estufas, la gestión pasa a estar mediada por una red experta, que es externa al hogar y distante de las prácticas tradicionales de cuidado.

\footnotetext{
“...la misma gente que te instala la estufa a pellet, te enseña los pasos básicos para usarla. Viene un manual, lo lees y tienes más o menos los funcionamientos de la estufa y eso. La programación es lo más complicado, en el fondo hay que leer más, estudiarlo, porque es programarlo, pero tampoco es tanta cosa, es como un reloj donde tienes que programar el lenguaje y poner la fecha, colocarle la hora para que funcione, digamos, bien". (Señor J, 2015).
}

Estas dificultades son reconocidas por los profesionales encargados del programa de recambio. Por ejemplo, en un estudio de seguimiento del uso de estufas se constata que un $14 \%$ de las familias tienen problemas de operar bien la estufa, un $8 \%$ por desprogramación de la estufa, un $8 \%$ por infiltraciones de la estufa y un $5 \%$ por dificultades para limpiar el artefacto ${ }^{4}$. Por consiguiente, este bloqueo visibiliza un aspecto de las tecnologías poco abordado, a saber, la centralidad de las redes e infraestructuras de mantención y cuidado en las cuales éstas descansan (Domínguez 2016). La incorporación de las nuevas estufas en una red profesional de mantención y cuidado opera como mecanismo de bloqueo a la transición, pues puede dificultar su uso en el tiempo, al limitar las posibilidades que tienen los usuarios de las nuevas estufas. Este hallazgo es consistente con una reflexión de larga data en los estudios de ciencia y tecnología relativas a lo determinante que es la fluidez y/o rigidez de las tecnologías en términos de su adopción (Laet y Mol 2000).

\section{DISCUSIÓN Y CONCLUSIONES: PENSANDO LAS POLÍTICAS ENERGÉTICAS DESDE LA SOCIOLOGÍA}

Los resultados de este estudio, indican que las transiciones energéticas a escala local son procesos de cambio complejos y multidimensionales que implican una gran variedad de actores.

El estudio revela que la aceptación y uso de las tecnologías energéticas emergentes en el sur de Chile depende de factores que están vinculados tanto a aspectos de diseño institucional, como a las prácticas sociales y materiales que se dan en el contexto doméstico. En las comunas de Temuco y Padre Las Casas existe una alta densidad de actores que tratan de incentivar la transición hacia formas más eficientes y menos contaminantes de calefacción. La política del recambio de estufas es un ejemplo de este tipo de intervención. Sin embargo, a pesar de los esfuerzos de la administración por acelerar el proceso de transición y proteger nichos tecnológicos emergentes, existen mecanismos de bloqueo que amarran la configuración del actual régimen socio-técnico. Por un lado, i) los problemas de coordinación entre diversas instituciones reguladoras y ii) las asimetrías de poder afectan a la creación de las condiciones externas necesarias para que los hogares adopten tecnologías alternativas a las estufas de leña. Por otro lado, hemos descrito como iii) las nuevas estufas implican una reconfiguración profunda de la ecología de prácticas asociadas a la calefacción. También, iv) estas sustituyen formas cotidianas de cuidado largamente establecidas en los hogares por un nuevo conjunto de prácticas mediadas por (nuevos) saberes expertos y sistemas técnicos de apoyo.

El concepto de bloqueo ha sido ampliamente utilizado desde enfoques institucionalistas en los estudios de difusión de nuevas tecnologías y configuraciones sociotécnicas. Los resultados de este trabajo muestran que es posible y fructífero trasladar esta noción a otras perspectivas teóricas, sin perder capacidad explicativa. Cuando analizamos la transición energética en ciudades del sur de Chile observamos que existen bloqueos operando a diferentes 
escalas. Además, la descripción de estos mecanismos de bloqueo en base al uso de las teorías MLP y TPS permite discutir algunas implicaciones tanto a nivel de políticas públicas como a nivel teórico. Por una parte, se observa que es necesario desarrollar formas de intervención sensibles a dificultades que operan a distintas escalas (desde el hogar, hasta el entramado institucional), evitando diseños que invisibilicen alguno de los niveles de análisis aquí descritos. Por otra parte, es también relevante estudiar la interrelación empírica entre los mecanismos descritos. Esta investigación se ha centrado en describir algunos mecanismos que frenan la aceptación y uso social de tecnologías energéticas emergentes en Temuco y Padre Las Casas, pero resulta difícil discernir el peso de cada uno de estos mecanismos y la relación entre ellos. En este sentido, algunas de las conclusiones del estudio deberán ser analizadas en profundidad y puestas a prueba como hipótesis de trabajo en futuras investigaciones.

En la línea propuesta por otros autores, nuestra investigación reivindica el papel de estudiar las transiciones energéticas a escala urbana (Rutherford y Coutard 2014; Mattes et al. 2015). Los estudios nacionales y globales que arrojan luz sobre las condiciones que pueden facilitar un cambio dirigido, sistémico y radical hacia unos patrones de provisión y uso de energía más sostenibles son de vital relevancia. Sin duda, hubiera sido interesante abordar con mayor profundidad el rol de la normativa y las políticas estatales en la transición energética. Pero el caso de la sustitución de las estufas de leña en Temuco y Padre Las Casas muestra que, de manera simultánea, las ciudades constituyen un foco de estudio clave. Los centros urbanos son entidades en las que se consume grandes cantidades de energía y en las que operan regímenes socio-técnicos que, hasta cierto punto, operan con unas reglas y una lógica interna propia, que no es rigurosamente trasladable a escala nacional. Por ese motivo (y por razones de espacio) han quedado fuera del análisis las interrelaciones entre las escalas nacional y local.

Por otra parte, en términos de los rendimientos de las teorías utilizadas, los resultados denotan que cuando se interrogan las políticas que incentivan la adopción de tecnologías energéticas en el hogar, es provechoso hacerlo con una mirada conciliadora, donde el enfoque de las transiciones energéticas y la teoría de las prácticas sociales consiguen dialogar. La raíz de los nudos que fondean los procesos de transición energética suele ser de diversa naturaleza, por lo que no parece conveniente que la investigación social se restrinja a un solo constructo teórico. La información aportada en este estudio muestra que elegir una u otra teoría para analizar una realidad social ontológicamente compleja, como es el caso de la contaminación atmosférica y transición energética de las ciudades intermedias del sur de Chile, nos aleja de una comprensión precisa del problema de investigación.

De hecho, las teorías MLP y TPS son complementarias en la medida que permiten identificar mecanismos de bloqueo que operan en escalas distintas. Mientras MLP visibiliza aspectos a nivel institucional que dificultan la transición (alta densidad de actores formales, problemas de coordinación entre instituciones y asimetrías de poder), la TPS hace visibles mecanismos de bloqueo situados en las prácticas y usos que, tradicionalmente un enfoque institucionalista dejaría de lado. En este sentido, y en línea con la literatura reciente (McMeekin y Southerthon 2012), ambas teorías se complementan dado que las unidades de análisis en que se centran son distintas. A esta relación entre teorías subsisten, sin embargo, ciertas tensiones. Por una parte, al separar marcos de análisis en escalas distintas, el uso complementario de las teorías propuesto en el artículo no permite evidenciar cual es el alcance de ambas teorías en la escala en que no se utilizan. Por ejemplo, desde la TPS sería posible estudiar la relación entre las prácticas tradicionales de calefacción y la constitución del entramado institucional, en la valoración que los actores institucionales hacen de la leña como un combustible "limpio". A su vez, desde una perspectiva MLP se podría estudiar cómo los mecanismos de bloqueo descritos a nivel de las prácticas se relacionan directamente con resistencias que se originan en el entramado institucional y las asimetrías de poder que vuelven difícil potenciar la transición en los hogares. Este tipo de limitaciones son propias de la adopción de un esquema analítico que organiza el alcance de estas teorías según escalas y requieren desarrollar formas de articulación empírica entre los distintos niveles (Crivits y Paredis 2013). A su vez, dan cuenta de las tensiones relativas a vincular marcos analíticos como MLP, que entienden los procesos de transición en términos de jerarquías, con aproximaciones como TPS cuya ontología social es de carácter relativamente simétrico (McMeekin y Southerthon 2012). Esto nos lleva a destacar otra de las limitaciones del estudio: la necesidad de problematizar la relación empírica entre las distintas escalas de análisis en las que operan los mecanismos de bloqueo. Tal reflexión es crucial para potenciar el diálogo entre ambas teorías, pero por razones de espacio no ha podido realizarse en el presente artículo.

\section{Agradecimientos}

Los autores agradecen el apoyo en terreno y análisis de la información de Fernanda Millán, Francisca Astorga, Boris Álvarez y Gabriela España, Juan Carlos Imio e Ignacio Orellana que ejercieron como ayudantes de investigación del proyecto. Este artículo fue financiado por el proyecto Fondecyt N¹1150262 y por la Iniciativa Científica Milenio, como parte del trabajo de Línea de Consumo Energético de NUMIES. 


\section{NOtAS}

1. Acrónimo que se otorga en Chile a las secretarías regionales ministeriales.

2. Cabe destacar que, en la mayoría de estudios empíricos, estos tres niveles se consideran independientemente de las escalas espaciales. No obstante, frecuentemente, los regímenes se atribuyen a nivel nacional.

\section{ReferenCias Bibliográficas}

Ariztía, T. 2017. "La teoría de las prácticas sociales: particularidades, posibilidades y límites". Cinta Moebio 59: 221-234. http://dx.doi.org/10.4067/S0717-554X2017000200221.

Bäfver, L. S., B. Leckner, C. Tullin y M. Berntsen. 2011. "Particle emissions from pellets stoves and modern and oldtype wood stoves". Biomass Bioenergy 35: 3648-3655. https://doi.org/10.1016/j.biombioe.2011.05.027.

Boardman, B. 2010. Fixing Fuel Poverty. Challenges and Solutions. London: Earthscan.

Boyatzis, R. E. 1998. Transforming qualitative information: Thematic analysis and code development. Londres y Nueva Delhi: Sage.

Braun V. y V. Clarke. 2006. "Using thematic analysis in psychology". Qualitative Research in Psychology 3: 77 101. http://dx.doi.org/10.1191/1478088706qp063oa.

Crivits, M. y E. Paredis. 2013.“Designing an explanatory practice framework. Local food systems as a case". Journal of Consumer Culture 13: 306-336. http://dx.doi. org/10.1177/1469540513484321.

Devine-Wright, P. 2005. "Beyond NIMBYism: towards an integrated framework for understanding public perceptions of wind energy". Wind energy 8: 125-139. http://dx.doi. org/10.1002/we.124.

Domínguez, F. 2016. "On the discrepancy between objects and things: An ecological approach". Journal of Material Culture 21: 59-86. http://dx.doi. org/10.1177/1359183515624128.

Fereday J. y E. Muir-Cochrane. 2006. "Demonstrating rigor using thematic analysis: A hybrid approach of inductive and deductive coding and theme development". International Journal of Qualitative Methods 5: 80-92. http:// dx.doi.org/10.1177/160940690600500107.

Foxon, T. J. 2002. 'Technological and institutional 'lock-in' as a barrier to sustainable innovation". London: Imperial College Centre for Policy and Technology (ICCEPT) Working Paper.

Frantzeskaki, N. y D. Loorbach. 2010. "Towards governing infrasystem transitions: reinforcing lock-in or facilitating change?" Technological Forecasting and Social Change 77: 1292-1301. http://dx.doi.org/10.1016/j. techfore.2010.05.004.

Geels, F. W. 2002. "Technological transitions as evolutionary reconfiguration processes: a multi-level perspective and a case-study". Research Policy 31: 1257-1274. http://dx.doi.org/10.1016/S0048-7333(02)00062-8.

Geels, F. W. 2004. "From sectorial systems of innovation to socio-technical systems: insights about dynamics and change from sociology and institutional theory". Research Policy 33: 897-920. http://dx.doi.org/10.1016/j. respol.2004.01.015.

Geels, F. W. 2005. "Processes and patterns in transitions and system innovations: refining the coevolutionary multilevel perspective". Technological Forecasting and So-
3. Por motivos de espacio obviamos en este artículo una descripción detallada del "Prendido de la estufa".

4. SIMCAM; Informe Final Licitación pública Id 608897-119Ip15, "Apoyo recambio de calefactores más eficientes y menos Contaminantes en viviendas de las comunas de Temuco y Padre Las Casas" 2016.

cial Change 72: 681-696. http://dx.doi.org/10.1016/j. techfore.2004.08.014.

Hine D., N. Bhullar, A. Marks, P. Kelly y J. Scott. 2011. "Comparing the effectiveness of education and technology in reducing wood smoke pollution: a field experiment". Journal of Environmental Psychology 31: 282-288. http://dx.doi.org/10.1016/j.jenvp.2011.05.003.

Instituto Nacional de Estadísticas. 2016. Compendio estadístico regional La Araucanía. Temuco: INE.

International Agency for Research on Cancer. 2010. Household Use of Solid Fuels and High-temperature Frying. Lyon: International Agency for Research on Cancer.

Kemp, R., J. Schot y R. Hoogma. 1998. "Regime shifts to sustainability through processes of niche formation: the approach of strategic niche management". Technology analysis \& Strategic management 10: 175-198. http:// dx.doi.org/10.1080/09537329808524310.

Klitkou, A., S. Bolwig, T. Hansen y N. Wessberg. 2015. "The role of lock-in mechanisms in transition processes: The case of energy for road transport". Environmental Innovation and Societal Transitions 16: 22-37. http://dx.doi. org/10.1016/j.eist.2015.07.005.

Krueger, R. A. y M. A. Casey. 2000. Focus Groups: A Practical Guide for Applied Research. 3a ed. Thousand Oaks, CA: Sage Publication.

Laet, M. y A. Mol. 2000. "The Zimbabwe bush pump: mechanics of a Fluid Technology". Social Studies of Science 30: 225-263. http://dx.doi. org/10.1177/030631200030002002.

Loorbach, D. 2010. "Transition management for sustainable development: A prescriptive, complexity-based governance framework". Governance 23: 161-183. http:// dx.doi.org/10.1111/j.1468-0491.2009.01471.x.

Loorbach, D. y J. Rotmans. 2006. "Managing transitions for sustainable development". Pp. 187-206 en Understanding Industrial Transformation: Views from Different Disciplines, editado por X. Olshoorn and A. J. Wieczorek. Dordrecht: Springer.

Mattes, J., A. Hube y A. J. Koehrsen. 2015. "Energy transitions in small-scale regions-What we can learn from a regional innovation systems perspective". Energy Policy 78: 255-264. http://dx.doi.org/10.1016/j.enpol.2014.12.011.

McMeekin, A. y D. Southerton. 2012. "Sustainability transitions and final consumption. Practices and socio-technical systems". Technology Analysis \& Strategic Management 24: 345-361. http://dx.doi.org/10.1080/0953732 5.2012 .663960

Ministerio de Desarrollo Social. 2013. "Encuesta de Caracterización Socioeconómica Nacional”.

Ministerio de Energía. 2015. Política de Uso de la leña y sus derivados para calefacción. Santiago de Chile: Ministerio de Energía. 
Ministerio del Medio Ambiente. 2016. Estado de calidad del aire por regiones del país y zonas de interés para alertas sanitarias. Seguimiento norma MP2.5, decreto $n^{\circ} 12$ del MMA. Santiago de Chile: Ministerio del Medio Ambiente.

Naeher L. P., M. Brauer, M. Lipsett, J. T. Zelikoff, C. D. Simpson, J. Q. Koenig y K. R. Smith. 2007. "Woodsmoke health effects: a review". Inhalation Toxicology 19: 67106. http://dx.doi.org/10.1080/08958370600985875.

Ochoa, R. G. y B. Graizbord. 2016. "Privation of energy services in Mexican households: an alternative measure of energy poverty". Energy Research \& Social Science 18: 36-49. http://dx.doi.org/10.1016/j.erss.2016.04.014.

Oltra, C., P. Upham, H. Riesch, À. Boso, S. Brunsting, E. Dütschke y A. Lis. 2012. "Public responses to CO2 storage sites: lessons from five European cases". Energy \& Environment 23: 227-248. http://dx.doi.org/10.1260/0958305X.23.2-3.227.

Pierson, P. 2000. "Increasing returns, path dependence, and the study of politics". American political science review 94: 251-267. http://dx.doi.org/10.2307/2586011.

Poumadère, M., R. Bertoldo, À. Boso, J. Espluga-Trenc, C. Mays, C. Oltra, A. Prades y N. Schneider. 2015. "Entre consommation durable et vie quotidienne: Les premiers balbutiements du compteur communicant en France et en Espagne". Pp. 403-419 en La Sociologie de l'énergie, editado por M. Beslay y C. Zélem. Paris: CNRS, Collection Alpha.

Rutherford, J. y O. Coutard. 2014. "Urban energy transitions: places, processes and politics of socio-technical change". Urban Studies 51: 1353-1377. http://dx.doi. org/10.1177/0042098013500090.

Sandén, B. A. y K. M. Hillman. 2011. "A framework for analysis of multi-mode interaction among technologies with examples from the history of alternative transport fuels in Sweden". Research Policy 40: 403-414. http://dx.doi. org/10.1016/j.respol.2010.12.005.

Schatzki, T. R. 1996. Social practices: A Wittgensteinian approach to human activity and the social. Cambridge: Cambridge University Press.

Shove, E. 2003. Comfort Cleanliness and Convenience. Oxford: Berg.

Shove, E. 2010. "Beyond the ABC. Climate change policy and theories of social change". Environment and planning A 42: 1273-1285. http://dx.doi.org/10.1068/a42282.

Shove, E., M. Pantzar y M. Watson. 2012. The dynamics of social practice: Everyday life and how it changes. London: Sage Publications.

Shove, E. y G. Walker. 2014."What Is Energy For? Social Practice and Energy Demand". Theory, Culture \& Society 31: 41-58. http://dx.doi. org/10.1177/0263276414536746.
Stern, P. 2014. "Individual and household interactions with energy systems: toward integrated understanding". Energy Research \& Social Science 1: 41-48. http://dx.doi. org/10.1016/j.erss.2014.03.003.

Strengers, Y. 2012. "Peak electricity demand and social practice theories. Reframing the role of change agents in the energy sector". Energy Policy 44: 226-234. http:// dx.doi.org/10.1016/j.enpol.2012.01.046.

Strengers, Y. y C. Maller. 2015. Social Practices, Intervention and Sustainability. Beyond behaviour change. London, New York: Routledge.

Unruh, G. C. 2002. "Escaping carbon lock-in". Energy Policy 30: 317-325. http://dx.doi.org/10.1016/S03014215(01)00098-2.

Upham, P., C. Oltra y À. Boso. 2015. "Towards a cross-paradigmatic framework of the social acceptance of energy systems". Energy Research \& Social Science 8: 100112. http://dx.doi.org/10.1016/j.erss.2015.05.003.

Verbong, G. P. J. y F. W. Geels. 2007. "The ongoing energy transition: lessons from a socio-technical, multi-level analysis of the Dutch electricity system (19602004)". Energy Policy 35: 1025-1037. http://dx.doi. org/10.1016/j.enpol.2006.02.010.

Verbong, G. P. J. y F. W. Geels. 2010. "Exploring sustainability transitions in the electricity sector with socio-technical pathways". Technological Forecasting and Social Change 77: 1214-1221. http://dx.doi.org/10.1016/j. techfore.2010.04.008.

Walker, G. 2014. "The dynamics of energy demand. Change, rhythm and synchronicity". Energy Research \& Social Science 1: 49-55. http://dx.doi.org/10.1016/j. erss.2014.03.012.

Walker, G., N. Simcock y R. Day. 2016. "Necessary energy uses and a minimum standard of living in the United Kingdom: Energy justice or escalating expectations?". Energy Research \& Social Science 18: 129-138. http:// dx.doi.org/10.1016/j.erss.2016.02.007.

Warde, A. y D. Southerton. eds. 2012. The Habits of Consumption (vol. 12). Helsinki: Helsinki Collegium in the Humanities and Social Sciences.

Welch, D. y A. Warde. 2015. "Theories of practice and sustainable consumption". Pp. 84-100 in Handbook of Research on Sustainable Consumption, editado por L. Reisch y J. Thøgersen. Edward Elgar Publishing.

Wilhite, H., H. Nakagami T., Masuda, Y. Yamaga y H. Haneda. 1996. "A cross-cultural analysis of household energy use behaviour in Japan and Norway". Energy Policy 24: 795-803. http://dx.doi.org/10.1016/03014215(96)00061-4. 
ÀLEX BOSO es Doctor por el Departamento de Teoría Sociológica, Filosofía del Derecho y Metodología de las Ciencias Sociales de la Universidad de Barcelona. Es profesor e investigador del Núcleo Científico Tecnológico en Ciencias Sociales y Humanidades de la Universidad de la Frontera, Temuco, Chile. Ha sido profesor asociado del Departamento de Ciencias Políticas y Sociales de la Universidad Pomeu Fabra y de la Facultad de Salud y Acción Social de la Universidad de Vic. Ha colaborado como investigador en el Centro de Investigación Sociotécnica del CIEMAT y en Internet Interdisciplinary Institute de la Universitat Oberta de Catalunya. Sus principales áreas de estudio son el análisis de las políticas públicas y las relaciones entre salud, medioambiente y migraciones. Sus trabajos han sido publicados en diversas revistas de relevancia académica, entre las que cabe destacar: Energy Policy; Energy and Environtment; Greenhouse Gases: Science and Technology; Energy Research \& Social Sicence, Basic Income Studies o Critical Social Policy. Actualmente dirige una investigación sobre procesos de implicación ciudadana en el control de la contaminación atmosférica de ciudades intermedias del sur de Chile.

TOMÁS ARIZTíA es Sociólogo. PhD en Sociología, London School of Economics and Political Science. Profesor Asociado de la Escuela de Sociología Universidad Diego Portales y Director del Magister en Métodos para la Investigación Social de esta misma casa de Estudios. Investigador Responsable Suplente del Núcleo Milenio en Energía y Sociedad (NUMIES). Sus intereses de investigación abarcan el entrecruce de tres áreas: la sociología del consumo sustentable, la sociología económica y la sociología del conocimiento. Ha publicado sobre estos temas en diversas revistas internacionales de sociología como el Sociological Review, Enviroment and Planning A, Journal of Consumer Culture y Journal of Cleaner Production. Actualmente coordina el área de "usos de energía" del Núcleo Milenio en Energía y Sociedad en la cual está realizando investigación etnográfica sobre las prácticas e infraestructuras de uso energético en los hogares y las dinámicas de transformación y comercialización de nuevas infraestructuras domesticas para el uso de energía.

FRANCISCA FONSECA es Socióloga. Doctora en Sociología por la Universidad Autónoma de Barcelona. Profesora Asistente de la carrera de Sociología y Directora del Departamento de Ciencias Sociales de la Universidad de La Frontera de Temuco Chile. Directora e investigadora del Centro de Investigaciones Sociales del Sur CIS-SUR de la Universidad de La Frontera. Investigadora Asociada del Núcleo Milenio en Energía y Sociedad (NUMIES). Sus principales áreas de investigación son: Sociología ambiental, Sociología del Riesgo Ecológico, Gobernanza Ambiental y de Recursos Naturales. Ha publicado sobre estos temas en revistas internacionales como: Revista de Sociología Papers, Revista Agua y Territorio. Actualmente dirige investigaciones sobre pobreza energética y producción energética en la Región de La Araucanía Chile. 